\title{
Exploitation of remote maintenance system based on constructional element
}

\author{
Lu Ping ${ }^{1}$, Li Min², Zhen Yunhui ${ }^{3}$ \\ ${ }^{1}$ Department of UAV Engineering, Ordnance Engineering College, Shijiazhuang 050003 China; \\ ${ }^{2}$ Joint Logistics Department of Hebei Provincial military command, Shijiazhuang 050083 China; \\ ${ }^{3}$ Hebei Provincial military, Shijiazhuang 050083 China \\ 2email: mahang1588@126.com
}

Keywords: remote maintenance system; components; domian analysis; reusable

\begin{abstract}
Based on the reusable components, this article developed a remote maintenance system software. Essence of the process is a reusable component extraction process and development. Under the guidance of the field of engineering analysis in the field of remote systems, identify reusable components, and achieve reusable components. Finally, remote maintenance systems software has been completed, can be used in other software. It saves development time and achieve program reusable.
\end{abstract}

\section{Introduction}

Remote repair is a measurement and control instrument network composed by the detection and diagnosis equipment. It is also a new repair method implemented multipoint communication network collaboration. This technology covers measurement and control instrument network formation, communication network construction, repair information system construction and so on. At present, the automatic testing of software reuse in the field of network is restricted to code reuse, rather than on the entire system structure reuse. Thus, developers must do a lot of work to handle control flow between objects in the system. Furthermore different developers often have different design for same project. The difference can lead to the specific architecture application of inconsistency, and result difficulties on maintenance and upgrading. Therefore, a reusable component development technology is needed on the development of software remote repair system, in order to make the reusing not only limited on the source code.

Component programming has been developed rapidly, when people realize the limitation of object-oriented method [1] [2]. Clements presents a component based software development (Component-Based Software Development, CBSD) concept. This concept formed the component based method of software engineering. This method changes the research and practice of software engineering from to a more accurate production scale with reuse of the code and component based software engineering method.

The software development process of component is consists two parts: the development of reusable components and use of reusable component development application system. This article focus on the first part of software development process of component, which is identifies the component elements in the field of reusable through the design of domain analysis, the remote repair system. UML modeling language was used during the field analysis phase and the field design stage in order to identify and extract reusable component in networked automatic test field. Part of the key components were actualized during implementation phase.

\section{Domain analysis for remote repair based on UML}

UML contains new theory, new method and new technology of software engineering field. It is not only applies to the support of object oriented analysis and design, but also supports the whole process of software development analysis. Therefore, the article develops domain analysis with UML modeling method process. Figure 1 shows the domain analysis process with UML method. 
The whole process is divided into three stages: the identifier field, analysis domain and the domain description. On the first stage, UML is used to make initial description for domain in order to determine the boundary and domain demand function; on the second stage, UML is used to describe the domain knowledge, object and its classification; on the third state, UML sequence diagram is used to describe the dynamic behavior of domain.

The corresponding UML modeling process can be summarized as follows: first, build use case model with demand analysis in order to get the function of the system; second, refine the use case model and establish static model of the system; finally, complete the function allocation between classes of the system, get class operation and establish the dynamic model of the system.

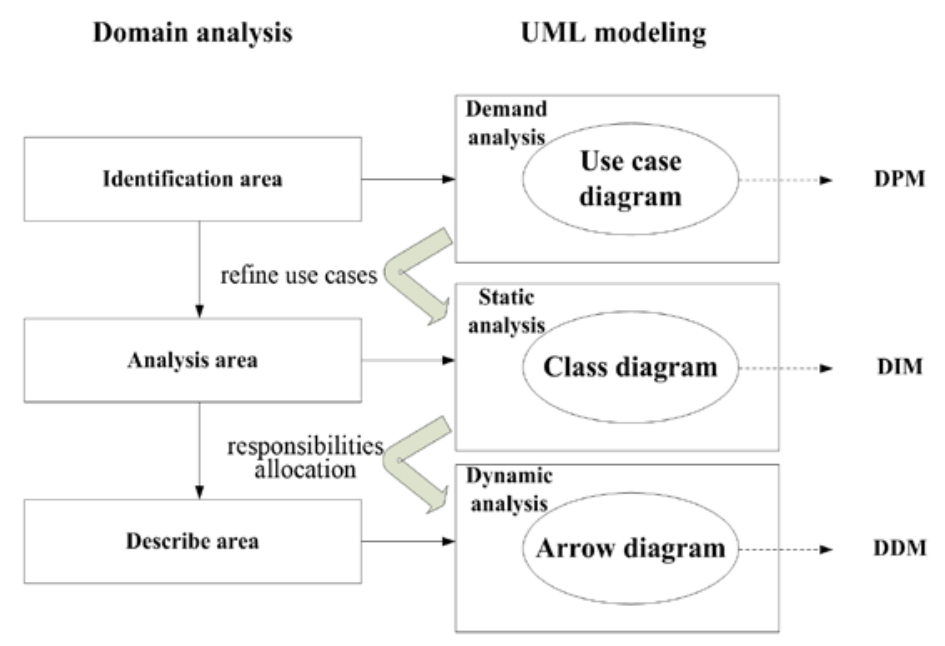

Figure 1 Domain analysis for remote repair based on UML

Case analysis is the most effective method that allows the developers to describe the user's requirements quickly and accurately. Using of case diagram is a high-level view to describe the system function from the user's point of view. Remote repair system uses case diagram to describe the system, to provide the function, and to inform the user how to use the system. The basic components of the case diagram includes the case roles and system.

Different use case diagrams were developed based on system software remote repair function model described above, according to different users. Use cases are used to describe the system function, also is the support should observe the system from user's perspective, helping analysts to understand the behavior of the system. Role is external entities interacting with the system, it can be a user of the system, and can also be other system or hardware equipment.

(1) The browser use case diagram

Figure 2 shows the browser use case diagram. User normally query tested information and diagnosis the fault through the browser page.

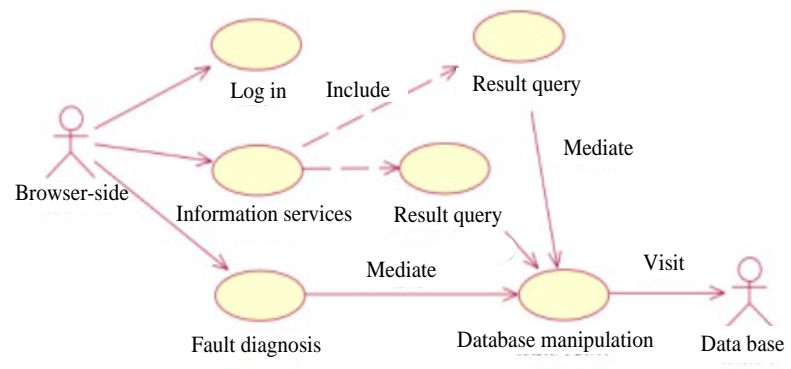

Figure 2 Browser use case diagram

(2) The client test executives use case diagram

Figure 3 shows the client test executive case diagram. The main users of remote repair system are the test executive. The main uses of test execution process of the system are self-checking, testing task management, execution, fault diagnosis, data management, and system synchronization management and system browsing that helps documentation. The testing task execution is the core function in the networked automatic test for remote repair system, and will be described in detail in 
this article. Data management includes the standardization of the data analysis, data description, data storage and query function.

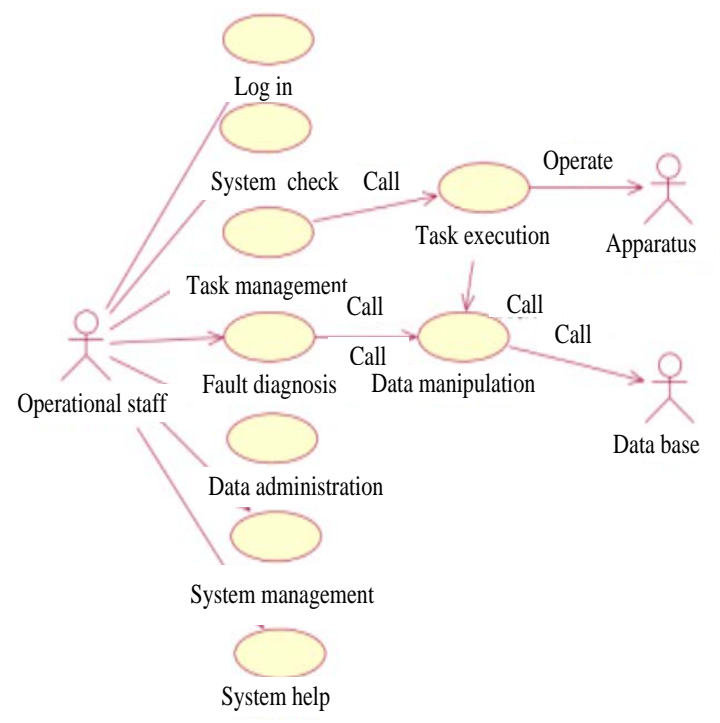

Figure 3 Client test executives use case diagram

(3) The system management use case diagram

Figure 4 shows the system administrators use case diagram, the system management functions are also implemented on the client. System management is mainly responsible for system management, maintenance work, includes user management, database management, network management, test equipment management and auxiliary function of management. The user management includes the addition of information management, system using personnel, users, new users log off expired on user permissions management; network management including networked automatic test for remote repair system based on the core of network connection management, test server or test equipment network management; management of test equipment management which including maintenance and update equipment, instrument driver management, instrument management and system configuration information description.

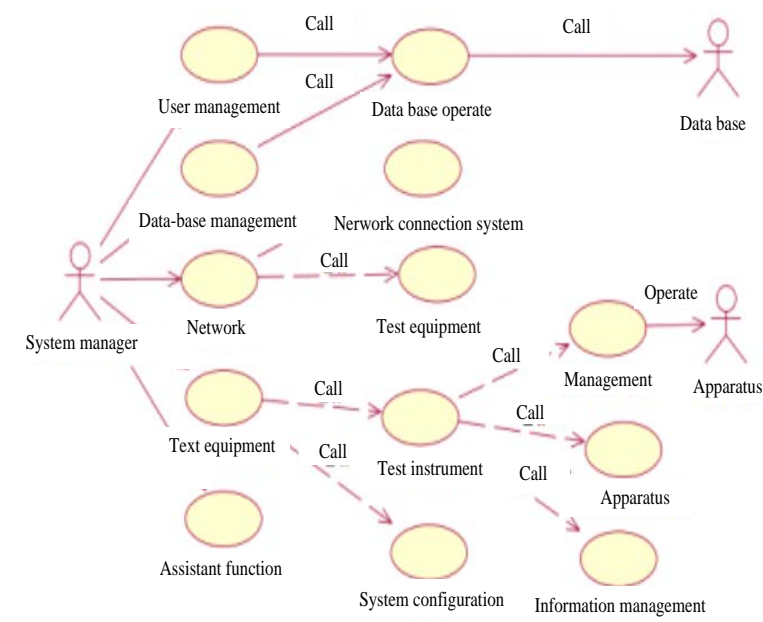

Figure 4 System management use case diagram

\section{Remote repair system field design}

Field design stage is to extent technology on the domain model, and distinguish the difference between general function points, option future points and difference points, based on the needs of domain. And use each generic function point as the field characteristics, build common abstract feature model based on those characters, and extract the reusable component, design the structure of software framework. 
The main task of domain design is extract the reusable component based on the analysis of the results of domain. The process that based on UML can be described as follows:

Step 1: Develop system function description through the use case diagram mapping system demand diagram. The analyst through the study of the use case diagram, can determine the responsibility of the problem domain and system. The problem domain here refers to the entire scope of business that need to be considered of the developed system;

Step 2: Describe the system's structure and function of the completion of the entity through the analysis of the static class diagram. Although the simple is differ from component in particle size and implementation, but it has same [package properties with component, and prepared a prototype for a component extraction

Step 3: Describe the dynamic collaboration relationship between the class object through the analysis of the sequence diagram. This step shows the extra or missing class more clearly.

Step 4: Develop the abstract component model of the system through the above analysis and combine the close related components based on the class diagram and actual demand. The relationships between classes include inheritance relationship, aggregation relationships and dependencies. The higher level on the use of the inheritance relationship should be considered In order to get the higher reusable member.

Component extraction process based on UML is an iterative process. The demand analysis, static analysis and dynamic analysis are mutually influence and interacts each other. The initial needs analysis can be carried out by static analysis and dynamic analysis, in the process of analysis, can capture requirements, extraction.

This project uses method mentioned above, completed within the field identification of component reuse auto test network, and build remote repair system based on component software structure diagram, shown in figure 3. According to the functional characteristics of components, this article hierarchically divided reusable component that identified from the process.

\section{The actualization of the field of remote repair system}

The main task of domain actualization is to identify reusable components through the design and implementation of domain analysis and domain design. The main topic of this section is remote repair system which includes remote object factory component and a resource manager component as example and introduction of its actualization process[3].

The remote object factory is use to (RemObjFactory) access the remote interface pointer from the resource manager component that distanced from test server during the test task execution process. The remote object factory contains an IRemObjFactory interface. The CreateRemObj method is for access the remote interface pointer from the resource manager component that distanced from test server, function parameter is used to specify a test server IP address, remote interface pointer output parameter $\mathrm{pVal}$ returns to the resource manager component, so that the test program can be dynamically assigned to the remote address of the test server.

The resource manager is uses (ResourceManager) to create various signal component object component mainly during the test task execution process. The factory method (FactoryMethod) model defines an interface for creating an object, and the interface is called the factory method.The purpose of factory method is to adapt changing of situation of created objects. In the remote repair system software platform for the signal, considering the need for the future upgrade of signal components, IResourceManager interface uses the factory method design resource manager component to complete the creation of various signal component objects.Users cannot create object interface signal components in the test. All signal components must be created by the IResourceManager interface. The greatest benefit is: when the signal component is updated or changed, only the IResourceManager interface need to be modified not the test program.

\section{Conclusion}

Component based software development changes the passive situation of the software 
development process and made it possible for the developer to purchase required components from market and assembled applications and made a revolutionary change in software industry. Later in other application development, the components can be used repeatedly, in order to save development time, achieve the reusing of test program.

\section{References}

[1] ZHANG Qi,CAO Jiang, ZHAO Hua zhang, etc.Implementation of engineering machine remote diagnosis and

maintenance system based on internet[J]. Journal PLA University of Science and Technology, 2009, 12: 47-52.

[2] WU Gong ping, HU Jie, HU Ji cai. Study and practice of equipment remote fault diagnosis system[J].Journal of Wuhan University, 2001, 34(2): 62-65.

[3] ZHANG Rong-tao, SUN Yu. Study on remote distributed intelligent monitoring diagnosis, maintenance system of project vehicle. China Mechanical Engineering. 2002, 13(12): 1028-1031. 